Cahiers Société

\title{
De la production au travail : à propos d'un changement de paradigme, ou comment Marx est devenu antiproductiviste
}

\section{Franck Fischbach}

Numéro 2, décembre 2020

Marx, critique du capital et de la société

URI : https://id.erudit.org/iderudit/1075559ar

DOI : https://doi.org/10.7202/1075559ar

Aller au sommaire du numéro

Éditeur(s)

Collectif Société

ISSN

2562-5373 (imprimé)

2562-5381 (numérique)

Découvrir la revue

Citer cet article

Fischbach, F. (2020). De la production au travail : à propos d'un changement de paradigme, ou comment Marx est devenu antiproductiviste. Cahiers Société,

(2), 205-228. https://doi.org/10.7202/1075559ar
Résumé de l'article

Nous défendons dans cet article la thèse selon laquelle l'évolution de sa pensée a conduit Marx à passer d'un paradigme philosophique à un autre, en l'occurrence du paradigme de la production à celui du travail. Nous cherchons à établir qu'il ne s'agit pas seulement du passage d'une pensée de la production à une pensée du travail, mais bel et bien d'une rupture dans la mesure où la philosophie du travail du dernier Marx est une philosophie explicitement antiproductiviste. Enfin, nous nous demandons quelles conséquences il est possible pour nous de tirer aujourd'hui, dans le contexte à la fois de crise du néolibéralisme et de crise écologique globale, de la thèse selon laquelle la position ultime de Marx a été celle d'une philosophie antiproductiviste du travail. 


\title{
De la production au travail : à propos d'un changement de paradigme, ou comment Marx est devenu antiproductiviste
}

\author{
Franck FISCHBACH \\ Université de Strasbourg
}

Nous nous proposons de défendre ici l'idée que l'évolution de sa pensée a conduit Marx à passer d'un paradigme philosophique à un autre, en l'occurrence du paradigme de la production au paradigme du travail. Nous chercherons donc à fonder ici la distinction entre un Marx 1, philosophe ou penseur de la production, et un Marx 2, philosophe ou penseur du travail. Cette proposition d'interprétation $\mathrm{du}$ parcours philosophique et théorique de Marx s'inscrit, on le voit, dans la suite déjà longue des lectures qui considèrent qu'il y a eu, à un certain moment, une rupture dans l'évolution de la pensée de Marx. Mais on voit aussi qu'il ne sera pas question ici de poser qu'une telle rupture aurait consisté, pour Marx, à passer de la philosophie à la science: il s'agit d'une rupture entre deux paradigmes également philosophiques, et non pas d'une rupture entre la philosophie et autre chose que la philosophie, que l'on peut appeler si l'on veut, avec Louis Althusser, science. Quant au moment où cette rupture avec le paradigme de la production serait survenue, il ne coïncide pas non plus avec le moment dans lequel Althusser a voulu voir le passage de la philosophie à la science. S'agissant en effet du rapport entre la production et le travail, L'idéologie allemande est un texte dans lequel il ne s'effectue aucun passage d'une prévalence de la première à un primat du second: au contraire, L'idéologie allemande peut certainement être considérée comme le texte qui illustre le mieux le paradigme productiviste qui a été celui de Marx avant que, bien plus tardivement - à la fin des années 1850 et au début des années 1860 - il ne passe définitivement au paradigme du travail et ne rompe avec le productivisme.

Dans notre tentative de justification de la distinction entre un Marx 1, penseur de la production, et un Marx 2, penseur du travail, nous commencerons par examiner les thèses d'auteurs qui, soit n'ont pas procédé à cette distinction et qui sont en conséquence conduits à reprocher à Marx de s'être contredit, soit ont proposé la distinction exactement inverse de la nôtre, opposant un jeune Marx qui aurait été un philosophe du travail et un Marx de la maturité qui serait devenu un théoricien de la production et un penseur productiviste. Nous justifierons ensuite notre propre distinction sur la base 
de l'examen d'un certain nombre de textes mêmes de Marx : nous verrons alors qu'il ne s'agit pas seulement du passage d'une pensée de la production à une pensée du travail, mais bel et bien d'une rupture dans la mesure où la philosophie du travail $\mathrm{du}$ dernier Marx ou de Marx 2 est une philosophie explicitement antiproductiviste. Enfin, nous nous demanderons quelles conséquences il est possible pour nous de tirer aujourd'hui, dans le contexte de crise du néolibéralisme et de crise écologique planétaire, de notre idée selon laquelle la position ultime de Marx a été celle d'une philosophie antiproductiviste du travail.

\section{Différentes façons de distinguer entre deux Marx}

Partons de la distinction que Arendt proposait de faire entre le travail (labour) et la production (work), dans la mesure où cette distinction supposait évidemment, de sa part, une explication avec Marx, à l'égard duquel elle reconnaît au demeurant une immense dette. En même temps, elle souligne le caractère équivoque, selon elle, de la position de Marx relativement au travail. Voici ce qu'elle écrit à ce sujet dans Condition de l'homme moderne :

L'attitude de Marx à l'égard du travail, c'est-à-dire à l'égard de l'objet central de sa réflexion, a toujours été équivoque. Alors que le travail est une «nécessité éternelle imposée par la nature », la plus humaine et la plus productive des activités, la révolution selon Marx n'a pas pour tâche d'émanciper les classes laborieuses, mais d'émanciper l'homme, de le délivrer du travail ; il faudra que le travail soit aboli pour que « le domaine de la liberté » supplante « le domaine de la nécessité ». [...] Des contradictions aussi fondamentales, aussi flagrantes sont rares chez les écrivains médiocres ; sous la plume des grands auteurs, elles conduisent au centre même de l'œuvre'.

Marx se serait en effet contredit s'il avait soutenu ces deux positions au sein d'une seule et même argumentation et au sein d'un seul et même paradigme. Or, ce n'est à notre avis pas le cas et, selon nous, Arendt ne voit ici de contradiction que parce qu'elle assimile les unes aux autres les positions que nous appellerons ici Marx 1 et Marx 2 : elle ne fait pas de distinction entre Marx 1, le Marx qui pense dans le cadre du paradigme de la production (le Marx des Manuscrits de 1844 et de L'idéologie allemande), et Marx 2, celui qui, à partir de la fin des années 1850, rompt avec ce paradigme productif et passe au paradigme du travail (c'est-à-dire le Marx des Théories sur la plusvalue et du Capital). Que le travail soit « une nécessité éternelle imposée par la

\footnotetext{
${ }^{1}$ Hannah Arendt, Condition de l'homme moderne, trad. G. Fradier, Paris, Press Pocket, p. 150-151.
} 
nature ${ }^{2} \gg$ est une position du Marx du Capital qu'on serait bien en peine de trouver dans L'idéologie allemande. Dans Le Capital, Marx pense en effet, comme le dit Arendt, que la tâche de la révolution est l'émancipation des classes laborieuses, et plus exactement l'auto-émancipation des classes laborieuses : c'est-à-dire que la tâche est que les classes laborieuses, la classe des travailleurs ou la classe ouvrière libère son activité de travail de son enrôlement par le processus capitaliste de valorisation indéfinie de la valeur. Il n'est pas question dans Le Capital de cesser de travailler ou d'abolir le travail, mais de travailler autrement (en l'occurrence : librement) et, pour cela, de transformer la société et les rapports sociaux afin que la force de travail ne soit plus la marchandise permettant l'extraction de la survaleur, et que les travailleurs ne soient donc plus sous la domination des propriétaires de capital.

En revanche, la position selon laquelle l'émancipation de la classe ouvrière est identique à l'émancipation de tous les hommes, parce que la première est « la représentante de toute la société », et l'idée que cela suppose de délivrer les hommes du travail - donc de supprimer la division sociale du travail, voire le travail lui-même - sont des positions et des idées propres à Marx 1, qu'il exprime en particulier dans L'idéologie allemande. Dans ce cas, en effet, il s'agit que les producteurs s'approprient l'ensemble des conditions sociales de la production et qu'ils libèrent la production sociale de la forme-travail en tant que cette forme impose à la société la division du travail et conséquemment la division en classes.

Il ne pouvait plus être question de cela dans Le Capital dès lors que le problème n'y est plus que la productivité humaine prenne la forme aliénante du travail, mais à l'inverse que le travail soit soumis au processus productif d'engendrement et de valorisation de la valeur. Dans le premier cas (donc pour Marx 1), il faut libérer la production de la forme-travail et, en un sens qui reste difficile à saisir, abolir la forme-travail ellemême ; dans le second cas (selon Marx 2), il faut libérer le travail de la forme productive, le libérer de son enrôlement par et de sa soumission à la productivité capitaliste : ce sont deux projets tout à fait différents dont les conséquences politiques sont elles aussi extrêmement différentes. Dans le premier cas, Marx 1 se place du point de vue de l'homme et il projette de le libérer du travail ; dans le second cas, Marx 2 se place résolument du point de vue des travailleurs, des hommes qui sont effectivement au travail (et qui le sont dans le cadre de rapports sociaux historiquement déterminés), il écrit une «économie politique du travail» contre «l'économie politique du capital », et, politiquement, il projette de libérer l'activité de ceux qui travaillent de la forme de production qui leur est imposée, qui les domine et qu'ils subissent.

On voit donc que la manière dont nous proposons de distinguer entre Marx 1 et Marx 2 s'oppose complètement à la façon dont d'autres avant nous ont conçu une

\footnotetext{
${ }^{2}$ La formule exacte est que le travail est « la condition naturelle éternelle de la vie des hommes » et que, à ce titre, il est « commun à toutes les formes sociales » (Karl Marx, Le Capital, livre 1: Le procès de production du capital, trad. J.-P. Lefebvre, Paris, Presses Universitaires de France, 1993, p. 207).
} 
distinction de ce genre. Jusqu'ici, soit on considérait (comme Arendt) que Marx a toujours dit à peu près la même chose sur la production et le travail (ou plutôt : qu'il est resté constamment dans la même ambiguïté à leur propos), de sorte qu'il n'y a pas lieu de distinguer entre des phases de sa pensée à ce sujet, soit on admettait qu'il y avait bien lieu de distinguer entre deux phases ou périodes s'agissant du travail et de la production, mais on les distinguait d'une façon exactement inverse de la nôtre. Ainsi, les expressions mêmes de «paradigme du travail» et de "paradigme de la production »-que nous utilisons à notre tour - proviennent d'un texte de Agnès Heller, sauf que cette dernière pensait que le paradigme du travail correspondait à la pensée du jeune Marx (ou Marx 1) et était particulièrement opérant dans un texte comme celui des Manuscrits économico-philosophiques de 1844 où selon elle, le travail est traité comme une constante anthropologique fondamentale, tandis que le paradigme de la production serait celui du Capital (donc Marx 2), où l'activité productive primerait sur toute autre forme d'activité3. Pour Heller, ce passage du paradigme du travail à celui de la production tiendrait au fait que Marx serait passé de la perspective d'une émancipation par le travail à celle d'une émancipation du travail, cette dernière étant inséparable du développement des forces productives et donc de celui du machinisme. Pour notre part, nous dirions que l'émancipation par le travail reste la perspective du dernier Marx, mais qu'elle ne lui apparaît désormais comme réellement envisageable qu'à la condition préalable que le travail lui-même soit d'abord libéré de sa captation et de son enrôlement par la production. Bref, selon nous, pour Marx 2, on ne pourra se libérer par le travail que lorsque le travail aura été libéré de la production, c'est-à-dire lorsque les travailleurs auront délivré leur activité de la soumission à toute forme d'impératif productif, comme tel hétérogène à leur activité propre (et faisant de celle-ci l'inverse d'une Selbsttätigkeit, d'une autoactivité).

La même conception se trouve aussi chez Anson Rabinbach : selon lui, Marx serait passé d'une conception selon laquelle « le travail est un chemin ou un mode de réalisation de l'homme dans son essence » à une conception qui revient à " considérer le travail comme une contrainte pesant sur la liberté4 $»$. En d'autres termes, Marx, à l'époque du Capital, «passe de l'émancipation de l'homme par le travail à l'émancipation $d u$ travail productif par une productivité supérieure, il devient "productiviste5" ». Où la formule « émancipation du travail productif» ne signifie pas délivrer ou libérer le travail de la production (ce qu'elle signifierait pour nous), mais se libérer du travail lui-même ou libérer les hommes du travail en transférant de plus en plus la production aux machines, en procédant au «remplacement progressif du travail humain par la technologie », et donc, selon l'expression de Rabinbach, en « réduisant le besoin de travail ${ }^{6} »$. Nous ne pouvons quant à nous manquer d'être surpris de l'usage

3 Agnès Heller, « Paradigm of production : paradigm of work », Dialectical Anthropology, vol. 6, 1981, p. 71-79. 4 Anson Rabinbach, Le moteur humain. L'énergie, la fatigue et les origines de la modernité, trad. M. Luxemboug et M. Cuillerai, Paris, La Fabrique, 2004, p. 128-129.

5 Ibid., p. 129.

${ }^{6}$ Idem. 
de cette expression de « réduction du besoin de travail » quand on se rappelle l'expression que Marx utilise en 1875 dans la Critique du programme de Gotha (donc Marx 2), à savoir que, dans la société libérée du capitalisme, le travail deviendra « le premier besoin de la vie » : cette perspective contredit expressément l'idée d'une réduction du besoin de travail.

La dernière proposition en date d'une distinction entre Marx 1 et Marx 2 au sujet du travail et de la production se trouve chez Thomas Coutrot7 qui oppose un « Marx $n^{\circ} 1$, celui de la liberté du travail » et un « Marx $n^{\circ}$ 2, progressiste et productiviste $^{8}$ ». « Marx $\mathrm{n}^{\circ} 1$ » est ici illustré par des passages empruntés notamment à la Critique du programme de Gotha où Marx invite à éliminer «l'asservissante subordination des individus à la division du travail et, par suite, l'opposition entre travail intellectuel et travail corporel», ce qui permettra que «le travail devienne non seulement le moyen de vivre, mais encore le premier besoin de la vie9 $»$. D'autres passages sont cités à l'appui de cette position qui serait donc celle de Marx 1, notamment un passage des Grundrisse où il est question d'un travail qui permettrait « une affirmation de liberté, la réalisation de soi, l'objectivation du sujet, donc sa liberté concrète qui s'actualise précisément dans le travail ${ }^{10} »$. Ce sont des textes qui vont dans le sens de l'idée d'un travail libéré ou d'un travail émancipé qui deviendrait le lieu même de la mise en œuvre et de l'expérience de la liberté.

Mais à ces textes s'opposent ceux représentatifs de la position de «Marx $n^{\circ} 2$ » : selon Marx 2, tel que Thomas Coutrot le comprend, " même débarrassé de l'emprise du capital, le travail ne pourra jamais être véritablement libre puisque sa finalité lui est imposée par les nécessités naturelles de la survie humaine, l'empire de la nécessité », de sorte que, « la vraie vie commençant au-delà du travail, l'émancipation passe donc en priorité par la réduction du temps de travail ». Et Thomas Coutrot de citer à l'appui le fameux passage du livre 3 du Capital où Marx pose que «la réduction du temps de travail est la condition fondamentale de [la] libération ${ }^{11} »$. D'où le fait que ce Marx 2 soit qualifié de "progressiste et productiviste» : pour réduire le temps de travail, Marx 2 s'en serait remis en effet au progrès technique tel qu'orchestré par le capitalisme lui-même, en particulier sous la forme du développement de la «machinerie » qui libère du temps pour les hommes, à mesure qu'il transfère du travail humain aux machines.

7 Thomas Coutrot, Libérer le travail. Pourquoi la gauche s'en moque et pourquoi ça doit changer, Paris, Seuil, 2018.

8 Ibid., p. 98-100.

9 Karl Marx, Critique du programme de Gotha, trad. S. Dayan-Herzbrun, Paris, Éditions sociales, 2008, p. 60 .

10 Karl Marx, Manuscrits de 1857-1858 (« Grundrisse »), t. 2, trad. J.-P. Lefebvre, Paris, Éditions sociales, 1980, p. 101.

${ }^{11}$ Karl Marx, Le Capital, livre 3 : Le procès d'ensemble de la production capitaliste, trad. C. Cohen-Solal et G. Badia, Paris, Éditions sociales, 1977, p. 742. 
Si Marx 1 et Marx 2 devaient être distingués à la manière dont Thomas Coutrot le propose, alors on serait contraint d'admettre qu'il y a non seulement, selon son expression, des «ambivalences de Marx sur le travail12 », mais même des contradictions, comme le pensait Arendt. Ce constat serait inévitable puisque Thomas Coutrot s'appuie sur des textes de Marx qui appartiennent tous à la période de pleine maturité de sa pensée, des Grundrisse au livre 3 du Capital et à la Critique du programme de Gotha. Le problème vient de l'attribution au Marx de la maturité d'une position productiviste qui n'est en réalité plus la sienne. C'est Marx 1 qui est productiviste, le Marx de L'idéologie allemande, et non pas le Marx du livre 3 du Capital, ni celui de la Critique du programme de Gotha. Le Marx tardif partisan de la réduction du temps de travail est au contraire celui qui cherche à libérer le travail de l'emprise de la production capitaliste, de sorte que, à l'inverse de ce que pense Thomas Coutrot, c'est bien plutôt Marx 2 qui est partisan de « libérer le travail », si on entend par là le fait d'aller vers des formes de travail qui soient synonymes de davantage de liberté effective et concrète pour ceux qui travaillent.

Notons ce qu'il y a de paradoxal de faire, comme Thomas Coutrot et Rabinbach avant lui, du Marx du Capital un penseur qui s'en serait remis au développement de la machinerie, tel qu'orchestré par le capitalisme lui-même, pour aller à la fois vers moins de travail et vers un meilleur travail. Le chapitre XIII du livre 1 du Capital dit exactement le contraire : Marx y explique le mécanisme en vertu duquel le développement de la machinerie a pour conséquence, sous le capitalisme, l'intensification de l'exploitation de la force humaine de travail13. Le Marx du Capital est celui qui explique que, non seulement le développement de la machinerie sous le capitalisme ne produit aucun effet libérateur sur les travailleurs, mais qu'il accentue au contraire leur exploitation, soulignant « ce phénomène remarquable dans l'histoire de l'industrie moderne ", à savoir que «c'est la machinerie qui fiche en l'air toutes les limites morales et naturelles de la journée de travail14 ». En d'autres termes, la machinerie en elle-même ne peut produire aucun effet libérateur aussi longtemps que persistent les rapports capitalistes de production : il faudrait d'abord être sorti de ces rapports pour que la machinerie puisse éventuellement produire un effet émancipateur.

Ainsi, contrairement à ce qu'affirme Thomas Coutrot, le travail "premier besoin de la vie» de la Critique du programme de Gotha ne s'oppose pas au « règne de la liberté » du livre 3 du Capital, au travail qui cesse d'être « dicté par la nécessité et les fins extérieures » et qui, « par sa nature même, se situe au-delà de la sphère de la production matérielle proprement dite 15 ». En effet, Marx ne dit pas que le « règne de la liberté » commence au-delà du travail, mais qu'il commence au-delà du travail « dicté par la nécessité et les fins extérieures » (je souligne) : c'est donc qu'une forme de

12 Thomas Coutrot, op. cit., p. 98.

13 Voir Karl Marx, Le Capital, livre 1, op. cit., p. 457.

14 Ibid., p. 458.

15 Karl Marx, Le Capital, livre 3, op. cit., p. 742. 
liberté commence avec le travail dès lors qu'il est organisé sur ses propres bases et se fixe à lui-même ses propres fins, au lieu qu'elles lui soient imposées par la nécessité naturelle et par les impératifs d'une "production matérielle » qui restent externes au travail et qui le contraignent de l'extérieur. Il y a une production matérielle à assurer en toute société, y compris dans la société post-capitaliste : une part du travail sera donc toujours non libre au sens où elle devra toujours être accomplie sous la contrainte des besoins à satisfaire, et donc par nécessité. Au-delà de cette part de travail imposée par la nécessité (qu'il est possible au demeurant d'accomplir « rationnellement», c'est-à-dire coopérativement, et de réduire - notamment grâce aux machines - mais pas d'éliminer), ce n'est pas le règne du non-travail (identifié au règne de la liberté) qui commence : il y a bel et bien encore du travail au-delà ou en plus du travail contraint par la nécessité naturelle, et il s'agit précisément du travail accompli librement. En ce sens, au-delà du travail que la contrainte des besoins impose d'accomplir, il y a les travaux auxquels on se consacre librement, sans y être contraint par aucune nécessité, et notamment pas par la nécessité naturelle. Il s'agit là d'un travail libéré de la contrainte, susceptible d'être accompli pour lui-même et de procurer plaisir et joie à ceux qui s'y consacrent en développant leurs facultés. Ainsi, quand Marx écrit que le « royaume de la liberté » commence avec «le développement des forces humaines comme fin en soi ${ }^{16} »$, il ne dit nulle part qu'un tel développement des forces humaines et un tel «épanouissement» n'ont plus aucun rapport avec le travail, encore moins qu'ils lui sont opposés : le déploiement d'une activité de travail voulue pour elle-même comme fin (et non plus comme moyen) peut parfaitement permettre le développement et l'épanouissement en question, et même leur être indispensable. Et lorsqu'il écrit que la condition de cet épanouissement est « la réduction de la journée de travail17 », il s'agit de la réduction dans la journée de la part de l'activité servant de moyen à la production des conditions de la vie.

En d'autres termes, il n'y a pas d'opposition entre Marx 1 et Marx 2 tels que Thomas Coutrot les distingue : le Marx qui veut réduire le temps de travail est très exactement le même que celui qui pense que le travail peut permettre le « développement des forces humaines » et leur épanouissement. Ce Marx-là ne pense pas que « la vraie vie commence au-delà du travail », pour la très simple raison que, d'une part, il n'y a pas de vie possible sans travail et que, d'autre part, il y a bel et bien des formes de travail qui relèvent de la «vraie vie », c'est-à-dire des formes de travail dans lesquelles il est possible de trouver un véritable accomplissement de soi - de sorte qu'il n'y a pas besoin d'être libéré du travail ou sorti du travail pour commencer à vraiment vivre et à vivre libre ${ }^{18}$. On peut même envisager que, une fois ces formes-là de travail

\footnotetext{
16 Idem.

17 Idem.

18 C'est un point qu'Alfred Schmidt avait très clairement aperçu. Il écrivait ainsi que, «pour Marx, le règne de la liberté ne remplace pas simplement le règne de la nécessité, mais le conserve en même temps en lui comme moment ineffaçable ». Il ajoutait « qu'une organisation plus rationnelle de la vie peut [selon Marx] certes limiter la durée du travail nécessaire à sa reproduction, mais ne peut pas entièrement
} 
devenues majoritaires, le travail devienne, comme Marx le dit dans la Critique $d u$ programme de Gotha, « le premier besoin de la vie ». Pour ce Marx-là, qui est celui que nous appelons Marx 2, il ne peut être question d'aller au-delà du travail, de surmonter le travail et encore moins de l'abolir : ces objectifs-là - qui n'étaient pas de maximiser le travail libre, mais de se libérer $d u$ travail - ont certes bien été ceux de Marx, mais il s'agit de Marx 1, celui en particulier de L'idéologie allemande qui dessinait en effet la perspective d'une abolition du travail dans une sorte de productivité sociale généralisée au sein de laquelle plus aucune forme d'activité ne s'isolerait, ni ne pourrait être isolée en tant que travail.

\section{Marx 1 : le paradigme de la production et la critique du travail aliéné}

Prétendre, comme nous le faisons ici, que Marx serait passé du paradigme de la production à celui du travail peut surprendre à plus d'un titre. Un premier motif de surprise réside dans le fait que l'un des textes les plus fameux du jeune Marx, les Manuscrits économico-philosophiques de 1844, soit connu comme le texte dans lequel Marx élabore une théorie du travail aliéné (die entfremdete Arbeit). On se dit alors que si le jeune Marx a pu élaborer une théorie du travail aliéné, ce ne peut être que dans la mesure où il était au même moment un théoricien ou un philosophe du travail, au sens où il a dû avoir une conception positive de ce qu'est ou de ce que devrait être le travail pour pouvoir faire porter sa critique sur les formes aliénées du travail - ce qui contredit immédiatement notre affirmation selon laquelle le paradigme philosophique du premier Marx aurait été celui de la production.

Afin de tenter de déterminer ce qu'il en est du rapport entre travail et production pour le jeune Marx, partons d'un passage significatif des Manuscrits de 1844 :

Car, tout d'abord, le travail, l'activité vitale, la vie productive n'apparaissent eux-mêmes à l'homme que comme un moyen en vue de la satisfaction d'un besoin, à savoir du besoin de conserver l'existence physique. Mais la vie productive est la vie générique. Elle est la vie qui engendre la vie. C'est dans la forme de l'activité vitale que repose le caractère entier d'une espèce, son caractère générique, et l'activité consciente et libre est le caractère générique de l'homme. La vie elle-même n'apparaît que comme un moyen de vivre'19.

supprimer le travail »; Marx, concluait Schmidt, « réconcilie la liberté et la nécessité sur la base de la nécessité » (Alfred Schmidt, Le concept de nature chez Marx, trad. J. Bois, Paris, Presses Universitaires de France, 1994, p. 183-184).

19 Karl Marx, Manuscrits économico-philosophiques de 1844, trad. F. Fischbach, Paris, Vrin, 2007, p. 122 (Marx, Engels, Werke, t. 40, Berlin, Dietz, 1990, p. 516 ; désormais MEW40). 
Le premier intérêt de ce passage est l'énumération de trois termes que Marx donne comme synonymes les uns des autres : le travail (die Arbeit), l'activité vitale (die lebendige Tätigkeit), la vie productive (das produktive Leben). Le travail est donc la même chose que l'activité productive et celle-ci est l'activité vitale de l'homme ou du genre humain : le travail ou l'activité productive est l'activité générique de l'homme en ce qu'elle est l'activité qui permet aux êtres humains de se maintenir en vie en tant qu'espèce - le travail ou l'activité productive est une activité vitale en ce sens-là, c'està-dire en tant qu'elle est une activité qui permet la vie, le maintien et la perpétuation de la vie. "La vie générique, aussi bien chez l'homme que chez l'animal, consiste d'abord physiquement en ceci que l'homme (comme l'animal) vit de la nature non organique $^{20} »$. Dire que l'humanité comme espèce vit de la nature non organique signifie que la nature et même, en l'occurrence, la totalité de la nature (puisque l'humanité est la seule espèce qui se rapporte à l'ensemble de la nature, et pas seulement à une partie ou à une région de celle-ci), est pour l'humanité comme son propre corps : dire, comme le fait Marx, que « la nature est le corps propre non organique de l'homme ${ }^{21}$ » signifie que l'humanité considère et traite l'ensemble de la nature comme son propre corps, bien que «la nature ne soit pas elle-même le corps humain ${ }^{22}$ ». La nature entière est le corps de l'humanité, un corps certes extérieur aux corps physiques des individus humains, mais un corps dans lequel ou au sein duquel les corps humains individuels sont contenus et dont ils sont eux-mêmes des parties. «Le fait que la vie physique et spirituelle de l'homme soit dépendante de la nature n'a pas d'autre sens que celui-ci : [...] l'homme est une partie de la nature $23 »$.

L'humanité comme genre doit en conséquence entretenir un processus constant (ständiger Prozess) avec la nature dont elle dépend de façon vitale puisqu'elle en est une partie : l'humanité ne peut pas ne pas avoir ni entretenir un tel rapport constant avec la nature puisque sa vie en tant que partie dépend de son rapport au tout. «L'homme vit de la nature signifie : la nature est son corps propre (Leib), avec lequel il faut qu'il demeure dans un processus continuel pour ne pas mourir 24 ». Et ce processus constant entre l'homme et la nature est assuré par le travail, c'est-à-dire par l'activité productive, mieux : ce processus constant d'interaction vitale entre l'homme et la nature est le travail lui-même, il est l'activité productive elle-même. Ce processus constant d'interaction homme/nature est donc aussi l'activité générique humaine comme telle : ce qui signifie que ce processus n'est pas un moyen dont l'humanité se servirait afin de se maintenir en vie. Ce processus est, pour l'humanité, sa vie même, et non pas le moyen de sa vie ou le moyen de se maintenir en vie.

\footnotetext{
20 Ibid., p. 121-122 (MEW40, p. 515).

21 Idem.

22 Idem.

23 Idem.

24 Idem.
} 
Ainsi donc l'activité productive, c'est-à-dire le travail, en tant qu'il est la forme que prend le processus constant d'interaction entre l'humanité et la nature dont elle dépend et dont elle est une partie, le travail donc est l'activité générique humaine en tant que telle, il est l'expression même et la plus propre de la vie humaine, la forme que prend nécessairement sa vie en tant qu'elle est la vie d'une partie de la nature, en tant que telle dépendante de son interaction avec le tout dont elle est une partie. Le travail est l'activité productive qui, dans l'interaction avec la nature et par « l'élaboration de la nature non organique $25 »$, rend possible «l'engendrement pratique d'un monde objectif $26 »$. Le sens de la production ou de l'activité productive réside entièrement dans cette « élaboration du monde objectif », c'est-à-dire dans le processus d'appropriation et de transformation de la nature qui résulte de l'interaction du genre humain avec le tout dont il est une partie. Mais, redisons-le, cette interaction n'est pas un moyen dont se doterait l'humanité, elle n'est pas un stratagème qu'aurait inventé l'humanité dans le but de se maintenir en vie : cette interaction et l'élaboration de la nature qu'elle permet sont les expressions de la vie même de l'humanité en tant que partie de la nature. C'est sa vie même et sa propre vie que l'humanité met en œuvre dans le travail : «la production est la vie générique [de l'homme] à l'œuvre » et «l'objet du travail est ainsi l'objectivation de la vie générique de l'homme27 ».

On le voit : il n'y a pas, pour le moment, dans la pensée de Marx, de distinction entre le travail et la production. Ils sont une seule et même chose. Mais passons maintenant à la question du travail aliéné, c'est-à-dire au travail rendu méconnaissable et étranger à lui-même. Là où le travail réalise l'interaction vitale de l'homme et de la nature, le travail aliéné accomplit au contraire la coupure et la séparation entre l'homme et la nature. Le passage du travail au travail aliéné consiste en une inversion complète du sens du travail. Alors que, du point de vue du travail, il apparaît que « le travailleur ne peut rien engendrer sans la nature, sans le monde extérieur sensible » et que, en ce sens, on peut dire que « la nature offre au travail son moyen de subsistance au sens où le travail ne peut pas subsister sans des objets à même lesquels il est exercé », inversement, du point de vue cette fois du travail aliéné, " plus le travailleur s'approprie par son travail le monde extérieur, la nature sensible, et plus il se soustrait de moyen de subsistance [...] en ce que le monde extérieur sensible cesse de plus en plus d'être un objet appartenant à son travail ${ }^{28}$ ».

Ce passage témoigne clairement du renversement qui se produit dans le rapport homme/nature lors du passage du travail au travail aliéné : d'une interaction entre l'homme et la nature ayant toutes les apparences d'une collaboration entre eux qui est telle que la nature fournit au travail humain les moyens dont il a besoin pour s'accomplir et les objets sur lesquels il s'exerce, on passe à une relation de conflictualité et

25 Ibid., p. 123 (MEW40, p. 516).

${ }^{26}$ Idem.

27 Idem (MEW40, p. 517).

28 Ibid., p. 119 (MEW40, p. 512-513). 
d'antagonisme consistant en ce que l'exercice du travail, la mise en œuvre de l'activité de travail a pour effet de lui soustraire dans la nature ses propres moyens de subsistance. Dans un cas, la nature « offre» au travail ce dont il a besoin pour s'effectuer, dans le second cas, le travail aliéné semble désormais s'effectuer contre la nature, l'appropriation qu'il réalise de la nature se traduit en soustraction des moyens de subsistance du travail lui-même. L'interaction collaborative entre l'homme et la nature se transforme en un rapport tel que le travail humain ne semble plus pouvoir se déployer que contre la nature et à son détriment : ce n'est plus la nature qui offre ses moyens au travail, c'est le travail qui dépossède la nature de ce dont il a besoin pour s'effectuer et que, désormais, il doit lui arracher parce qu'elle ne les lui offre plus et, au contraire, les lui soustrait.

Là où, dans le travail et l'activité productive, il y a continuité entre le travail humain et la nature, continuation de la seconde par le premier, il y a en revanche, dans le travail aliéné, rupture entre l'homme et la nature. Et cette rupture de l'interaction entre l'homme et la nature est le présupposé de toute l'analyse que donne Marx du travail aliéné. En ce sens, dans les Manuscrits de 1844, l'opposition pertinente n'est pas encore entre travail et production, mais entre, d'une part, le travail et la production et, d'autre part, le travail aliéné. Le travail et la production relèvent tous les deux de ce que Marx nomme à l'époque «l'industrie humaine » dont le développement apparaît comme étant entravé par la forme aliénée du travail. L'aliénation du travail est un blocage de la production, c'est-à-dire de l'industrie. L'aliénation est vue comme une perturbation injustifiée de la production, qui vient rompre la continuité entre le développement de l'industrie humaine et l'affirmation de la productivité de la nature : en venant rompre le lien entre le travail humain et ses conditions objectives, c'est-àdire naturelles - un lien qui est condition de possibilité du déploiement de l'industrie -, l'aliénation vient gâcher les possibilités d'affirmation de soi des hommes dans et par leur industrie ; elle entrave les chances d'un déploiement de l'industrie humaine en continuité, voire en collaboration avec la productivité naturelle.

Lorsque, des Manuscrits de 1844, on passe à L'idéologie allemande, on ne peut qu'être frappé par les profondes transformations intervenues dans la pensée de Marx. La plus significative consiste sans doute en la séparation des concepts de travail et de production: pour le dire vite, nous avons affaire à une promotion du concept de production et à une dévalorisation concomitante du concept de travail. Cela se remarque d'abord à l'apparition des thématiques nouvelles de «l'abolition (Aufhebung) du travail » et de «l'abolition de la division du travail». Ces thèmes apparaissent notamment dans un passage où Marx pose que les individus sont subsumés sous leur classe de la même façon qu'ils sont subsumés sous la division du travail : dans les deux cas, les individus sont déterminés par des conditions qu'ils trouvent déjà là. Ils « se voient dicter leur position dans la vie » du fait d'une appartenance de classe dont ils ne décident pas, et, de même, ils se voient dicter le type de leur activité par la division du travail dont ils ne décident pas davantage que de leur appartenance de 
classe : Marx pose que « la subsomption des individus singuliers sous la division du travail » est « le même phénomène » que leur subsomption sous la classe, et il ajoute qu'elle « ne peut être supprimée que par l'abolition (die Aufhebung) de la propriété privée et du travail lui-même 29 ». La propriété privée qui apparaît ici relève de ce que Marx commence, dans L'idéologie allemande, à appeler les rapports de production : elle est une forme de ces rapports qui apparaît comme une entrave au plein développement des forces productives ${ }^{\circ}$, de même que le travail apparaît ici comme une activité dont la forme maintient les travailleurs séparés des forces productives et donc séparés d'eux-mêmes. "Les forces productives, écrit Marx, ne connaissent sous la propriété privée qu'un développement unilatéral, elles deviennent pour le plus grand nombre des forces destructives ${ }^{31} \gg$. Ce retournement des forces de production en forces de destruction sous le régime de la propriété privée se manifeste en particulier dans le fait que, comme dit Marx, "le travail lui-même devient insupportable au travailleur $32 »$. En adoptant la forme du travail, l'activité productive du travailleur, qui devrait consister en une affirmation de lui-même et en ce que Marx appelle une « autoactivité » (Selbsttätigkeit), se renverse en son exact contraire : elle devient une force de destruction du travailleur individuel.

On comprend mieux dans ces conditions que l'objectif puisse pour Marx être celui de l'abolition du travail, c'est-à-dire l'abolition de la forme travail de la production. Plusieurs thématiques se croisent ici : d'une part, celle qu'on vient de voir, le fait que le travail soit souffrance là où la production devrait être affirmation et jouissance, mais aussi, d'autre part, le fait que la forme-travail impose une division des activités humaines entre celles qui sont posées et reconnues comme relevant du travail, et celles qui ne le sont pas, en même temps qu'elle assigne l'individu à une seule forme de travail dans l'ignorance et l'abstraction de toutes les autres - ce qui a pour conséquence de laisser en jachère toutes les potentialités productives qui sont celles d'un individu, et donc aussi celles de la société dans son ensemble. D'où ce très fameux passage :

Aussitôt que le travail commence à être réparti, chacun a un cercle d'activité exclusif et déterminé qui lui est imposé et dont il ne peut sortir ; il est chasseur, pêcheur ou berger, ou critique critique, et il doit nécessairement le rester s'il ne veut pas perdre les moyens qui lui permettent de vivre - tandis que dans la société communiste, où chacun n'a pas un cercle d'activité exclusif, mais peut se former dans n'importe quelle branche, la société règle la production générale et, de ce fait, m'offre la possibilité de faire aujourd'hui ceci, demain cela, de chasser le matin, de pêcher l'après-midi, de

\footnotetext{
29 Karl Marx, Friedrich Engels et Joseph Weydemeyer, L'idéologie allemande. $1^{\text {er }}$ et $2^{e}$ chapitres, trad. J. Quétier et G. Fondu, Paris, Éditions sociales, 2014, p. 195.

30 La propriété privée est « une chaîne » pour « la masse de forces productives » engendrée par la grande industrie (ibid., p. 183).

${ }^{31}$ Idem.

32 Ibid., p. 185.
} 
pratiquer l'élevage le soir et de critiquer après le repas, exactement comme j'en ai envie, sans jamais devenir chasseur, pêcheur, berger ou critique33.

Ce passage s'articule autour d'une relation d'opposition entre le travail et la production : d'un côté, il y a le travail divisé et réparti qui consiste en l'assignation à chacun d'un " cercle exclusif d'activité », tandis que de l'autre côté - qui fait l'objet d'une évaluation normative positive de la part de Marx-, on trouve la "production générale » qui est socialement régulée. Du côté de la production générale, on a une unité de l'activité individuelle particulière et de l'intérêt commun universel dans la mesure où les activités de production sont socialement régulées, tandis que du côté $d u$ travail divisé, on a au contraire une séparation et une opposition entre l'activité particulière de travail et l'intérêt universel ou général : ce dernier reste extérieur à l'activité individuelle de travail et s'impose à elle de l'extérieur sous la forme d'une contrainte qui est d'abord celle de la dépendance réciproque entre toutes les activités individuelles de travail, dont chacune subit ainsi l'ensemble de toutes les autres en tant que l'ensemble des conditions dont elle dépend et qu'elle ne maîtrise pas. C'est pourquoi Marx note que, « avec la division du travail est en même temps donnée la contradiction entre l'intérêt de l'individu singulier ou de la famille singulière et l'intérêt communautaire de tous les individus qui ont commerce les uns avec les autres » - cet intérêt communautaire existant d'abord «dans la réalité effective en tant que dépendance réciproque des individus entre lesquels le travail est divisé34 ».

C'est là le propre d'une société du travail divisé, c'est-à-dire d'une société qui ignore l'idée d'une production socialement régulée : l'intérêt même de la société comme tel, que Marx appelle «l'intérêt communautaire », n'y peut prendre que la forme d'une régulation externe, formelle et aveugle qui contraint chaque activité séparée de production - qui, en tant que séparée, prend la forme d'un travail - du poids de toutes les autres activités de production avec lesquelles la première est obligée d'entretenir un commerce afin de pouvoir s'accomplir. Et c'est là la raison pour laquelle,

aussi longtemps que l'activité n'est pas le fruit de la volonté libre, mais qu'elle est divisée de façon spontanée (naturwüchsig), l'acte propre de l'homme devient pour lui une puissance étrangère qui lui fait face, qui le place sous son joug, au lieu que ce soit lui qui domine cet acte35.

Le travail est ainsi le nom que prend l'activité productive quand elle cesse pour chacun d'être son activité propre et devient l'activité coagulée de tous les autres qui viennent contraindre et éventuellement contrecarrer la sienne propre de l'extérieur : en ce sens,

33 Ibid., p. 77-79.

34 Ibid., p. 77.

35 Idem. 
le Marx de L'idéologie allemande est celui qui pense qu'il n'y a plus de travail qu'aliéné, ou que le travail est le nom qui convient à l'activité productive quand elle est aliénée, c'est-à-dire quand elle n'est plus pour personne son activité propre, son « auto-activité » (Selbsttätigkeit), mais le résultat coagulé, contraint, limité et borné de l'activité anonyme de tous les autres. Aussi Marx peut-il alors écrire que

chez les prolétaires, leur propre condition de vie, le travail, ainsi que toutes les conditions d'existence de la société actuelle sont devenues pour eux une chose contingente sur laquelle les prolétaires singuliers n'ont aucun contrôle [...], et la contradiction entre la personnalité du prolétaire singulier et la condition de vie qui lui est imposée, le travail, se fait jour pour lui-même36.

Le travail est ainsi la « condition de vie » qui est imposée aux travailleurs, une condition qui vient contredire leur " personnalité » dans la mesure même où le travail est directement contraire et s'avère un obstacle au déploiement de l'auto-activation, c'està-dire à l'affirmation de ce qui est constitutif de la personnalité. Il ne peut donc s'agir que de se libérer du travail, ce qui suppose selon Marx « la transformation du travail en auto-activation », de sorte que « l'auto-activation coïncide avec la vie matérielle37 », c'est-à-dire avec la production sociale et matérielle. Et si la spécificité du moment historique dont Marx est le contemporain consiste en ce que « les forces productives se sont développées jusqu'à former une totalité » et en ce qu'elles «n'existent qu'à l'intérieur du commerce universel », alors Marx estime pouvoir dire que

seuls les prolétaires d'aujourd'hui, qui sont complètement exclus de toute auto-activation, sont en mesure d'imposer leur autoactivation complète et non plus bornée, laquelle consiste dans l'appropriation d'une totalité de forces productives et dans le développement d'une totalité de facultés que celle-ci implique38.

En d'autres termes, contre le travail qui sépare les prolétaires de toute possibilité d'auto-activation, il faut procéder à un renversement complet, comme tel révolutionnaire, qui consiste en un passage brutal à l'auto-activation par l'appropriation de la totalité des forces productives ou des forces productives totalement développées : se libérer du travail, forme bornée et entièrement subie d'hétéro-activation, revient pour les travailleurs à entrer dans un devenir productif total qui coïncide avec leur autoactivation. L'appropriation totale de forces productives elles-mêmes totalement développées, le devenir totalement productif des prolétaires dans une société elle-même intégralement productive, telle est donc la manière dont le Marx de L'idéologie

\footnotetext{
${ }^{6}$ Ibid., p. 203.

37 Ibid., p. 233.

38 Ibid., p. 231.
} 
allemande conçoit qu'il soit possible de se libérer du travail. On sort du travail par la production et en faisant coïncider la production avec l'auto-activation.

\section{Marx 2 : le paradigme du travail et la critique de ce que la production fait au travail}

Venons-en maintenant à la position du dernier Marx ou de Marx 2. Cette position est notamment exprimée en 1864 dans le Manifeste inaugural de l'Association Internationale des Travailleurs. Marx pose ici clairement que l'objectif est, selon sa propre expression, « l'émancipation du travail », et il donne deux exemples des progrès accomplis en direction de cette émancipation dans les années qui précèdent son Manifeste inaugural. Le premier exemple est celui de la « limitation légale des heures de travail » et de la conquête du bill des dix heures par les ouvriers anglais : dans la lutte pour l'obtention de cette limitation légale de la durée du travail, c'était aussi «l'économie politique de la classe ouvrière » qui affrontait «l'économie politique de la bourgeoisie »; c'était « la grande querelle entre le jeu aveugle de l'offre et de la demande, qui est toute l'économie politique de la classe bourgeoise, et la production sociale contrôlée et régie par la prévoyance sociale, qui constitue l'économie politique de la classe ouvrière », et, " pour la première fois au grand jour », c'est " l'économie politique du travail » qui a remporté une victoire à la fois pratique et symbolique sur «l'économie politique de la propriété ».

Le second exemple que donne Marx du progrès vers l'émancipation du travail est celui « du mouvement coopératif et des manufactures coopératives » : là encore, c'est la victoire d'un principe sur un autre, en l'occurrence celle du travail libre et autonome sur le travail dominé et asservi à une production dont les instruments et les finalités lui échappent. La valeur de ces « grandes expériences sociales » que sont les coopératives tient à ce que, selon Marx, elles ont démontré « qu'il n'était pas nécessaire pour le succès de la production que l'instrument de travail fût monopolisé et servît d'instrument de domination et d'extorsion contre le travail lui-même », elles ont montré que, " comme le travail esclave, comme le travail serf, le travail salarié n'était qu'une forme transitoire et inférieure, destinée à disparaître devant le travail associé ». Les deux exemples mobilisés par Marx illustrent l'idée du travail posé comme un principe sur la base duquel peut s'opérer une transformation de la production : c'est le travail émancipé de la soumission à la production et devenant maître de celle-ci, à la fois parce que c'est lui qui limite le temps consacré à la production et parce que c'est lui qui décide de la forme de production en lui imposant celle de la coopération et de l'association.

On comprend dès lors que Marx puisse interpréter sa propre entreprise théorique comme étant celle d'une critique de l'économie politique bourgeoise qui est faite du point de vue de ce qu'il appelle lui-même « l'économie politique du travail ». Ce point de vue est très clairement celui adopté par Marx dans Le Capital. 
Dans le chapitre V du livre 1 du Capital, Marx donne une première définition du travail productif du point de vue du « procès de travail », celui-ci étant lui-même saisi abstraitement, c'est-à-dire "indépendamment de toute forme sociale déterminée 39 ». Saisi indépendamment des formes de sociétés dans lesquelles il s'inscrit à chaque fois, le procès de travail est lui-même composé de trois "moments simples ${ }^{40} »$ : d'abord l'activité que déploie un individu en vue de la fin qu'il se propose de réaliser (cette activité individuelle consciente orientée vers une fin étant ce que Marx appelle «le travail proprement dit41 »), ensuite l'objet sur lequel porte ou bien auquel s'applique cette activité qu'est le travail, et enfin le moyen qu'utilise le travail pour réaliser sa fin. L'objet de travail, c'est ce que l'individu trouve comme étant déjà là : c'est l'ensemble des choses que « la terre » fournit à l'homme et que « le travail n'a qu'à détacher de leur liaison immédiate avec le tout terrestre $42 »$. Le moyen de travail, c'est ce que l'individu « insère entre son objet de travail et lui43». Le moyen de travail suppose un acte de l'individu, de sorte que si l'objet de travail est ce que l'individu « trouve tel quel par nature », le moyen de travail est ce dont l'individu " s'empare immédiatement 44 » et qui ne prend sens non pas comme ce à quoi son activité s'applique, mais comme ce qui permet et prolonge cette activité, il est ce dont l'individu s'empare pour en faire « un organe de son activité45 ». De cette manière, et si donc, comme dit Marx, « on considère l'ensemble de ce procès du point de vue de son résultat, [à savoir] le produit, [alors] moyen de travail et objet de travail apparaissent l'un et l'autre comme des moyens de production, et le travail lui-même comme travail productif $46^{\prime}$ ». C'est évidemment au regard de son produit que le travail peut se définir comme productif, et le produit est ici « une valeur d'usage, une matière naturelle appropriée à des besoins humains par une modification de sa forme $47 \gg$.

L'ensemble du procès de travail nous amène donc à une compréhension du travail productif en tant qu'activité que déploie un individu en vue d'une fin qu'il se représente consciemment ; cette fin étant en l'occurrence d'engendrer un objet utile, c'est-à-dire un objet qui satisfasse un besoin humain, de sorte que l'activité visant cette fin consiste à " provoquer une modification de l'objet de travail grâce au moyen de travail $48 »$.

On a là une première approche du travail productif ou, plutôt, de ce que Marx appelle plus précisément le travail «immédiatement productif» (je souligne). Au

\footnotetext{
39 Karl Marx, Le Capital, livre 1, op. cit., p. 198.

40 Ibid., p. 200.

${ }^{41}$ Idem.

42 Ibid., p. 201.

43 Idem.

44 Idem.

45 Idem.

46 Ibid., p. 203.

47 Idem.

48 Idem.
} 
regard de cette première approche ou de cette saisie immédiate qui, en tant que telle, ne peut être qu'abstraite et générale, on peut dire qu'il n'y a pas de travail qui ne soit productif : tout travail consiste en la mobilisation par un individu donné de son activité propre en vue de la fin qui consiste à produire une chose utile, et à le faire en utilisant des moyens qui permettent de modifier un objet trouvé en lui donnant une forme qui le rende précisément utile à la satisfaction d'un besoin. Le problème est que ce travail immédiatement productif n'existe comme tel en réalité nulle part pour la raison qu'un procès de travail vient toujours prendre place au sein d'une formation sociale, au sein d'une organisation sociale qui lui donne à chaque fois une forme particulière. C'est pourquoi, lorsque Marx rappelle cette première définition du travail productif au seuil du chapitre XIV ( Survaleur absolue et relative »), il rappelle également la note 7 qui figure dans le chapitre $\mathrm{V}$ dans laquelle il précise que « cette définition du travail productif, telle qu'elle ressort de la perspective du procès de travail simple, ne suffit absolument pas pour le procès de production capitaliste49 », pas plus d'ailleurs qu'elle ne suffit pour aucun autre procès de production à partir du moment où celui-ci est inscrit dans une formation sociale déterminée.

Cela tient au fait que le procès de travail n'a jusqu'ici été abordé que comme un procès individuel, comme un procès consistant en l'interaction d'un individu avec les objets naturels qu'il trouve et qu'il se fixe pour fin de transformer en des produits utiles. Comme le dit Marx, " dans l'appropriation individuelle des objets de la nature à ses propres finalités vitales, [l'individu] a le contrôle de lui-même [;] c'est plus tard qu'il est contrôlé5o », et il l'est ou le devient en l'occurrence par la société dans laquelle vient prendre place son activité de travail productif. Dans l'analyse du procès de travail, tout change donc quand on introduit la perspective sociale en lieu et place de la perspective strictement individuelle qui a été jusqu'ici retenue. Ainsi, poursuit Marx, « de même que le système de la nature fait de la tête et de la main un ensemble unique, le procès de travail réunit travail cérébral et travail manuel [;] c'est par la suite qu'ils se dissocient et finissent même par s'opposer comme des ennemis ${ }^{1} »$. Ainsi donc, il en va de même du contrôle de l'activité individuelle et de la dissociation des aspects manuels et intellectuels de l'activité de travail : ils n'apparaissent que du fait de l'inscription de cette activité dans une formation sociale donnée et ils ne se manifestent pas aussi longtemps qu'on ne prend sur l'activité de travail qu'un point de vue individuel, non historique, non social et donc abstrait.

Dès qu'on introduit l'élément social et historique, on introduit aussi la division du travail interne à l'organisme social : l'activité individuelle de travail apparait alors comme le résultat de la division d'une activité globale de travail qui est celle de l'ensemble de la société, et les aspects qui étaient unis dans l'activité individuelle de travail se séparent à leur tour, l'activité manuelle se sépare de l'activité intellectuelle

\footnotetext{
49 Ibid., p. 203 et p. 569.

50 Ibid., p. 569.

${ }^{1}$ Idem.
} 
et elles échoient à des individus et à des groupes différents d'individus. Mais il arrive aussi qu'il n'y a plus de rapport direct et donc immédiat entre le producteur individuel et le produit de son activité de travail : la production du résultat de l'activité de travail suppose l'intervention d'une multiplicité d'autres producteurs, ceux qui ont déjà élaboré avant lui ce qui devient l'objet de son travail, par exemple, les fileurs qui fournissent son fil à celui qui va faire la toile, mais aussi ceux qui ont produit les outils qu'il utilise, mais encore ceux dont l'activité est requise dans l'élaboration du même produit à un autre stade de sa fabrication. Bref, « le produit se transforme en produit social collectif d'un travailleur global52 ».

On voit alors que « c'est la notion même de travail productif, ou de son porteur, la notion de travailleur productif, qui s'étend $53 »$. Elle s'étend en ce que n'est plus considéré comme productif le seul individu qui est directement et immédiatement en relation avec l'objet du travail ou celui qui, comme dit Marx, " met lui-même la main à la pâte »: plus besoin, pour être travailleur productif, d'être à la fois celui qui conçoit l'objet à produire, qui utilise l'objet du travail et lui applique les moyens du travail. Il suffit désormais, pour être un travailleur productif, d'être impliqué dans l'une ou l'autre des étapes du procès qui conduit au produit: « il suffit d'être un organe quelconque du travailleur global et d'exécuter l'une de ses sous-fonctions 54 ». En ce sens, celui qui prend part à la conception de l'objet à produire est tout aussi productif que celui qui implique directement son énergie physique dans le procès de la production, ce dernier ne devant plus, pour être productif, avoir de rapport avec l'objet fini, son intervention à l'un des stades de la production suffisant amplement à le rendre productif.

On a donc une diffusion du travail productif qui est telle que ce travail s'étend à la société entière et que celle-ci devient un travailleur global, tous les individus participant à un titre ou à un autre de cette activité sociale globale de production devenant eux-mêmes des travailleurs productifs. La socialisation du travail, c'est-à-dire la socialisation des objets, des moyens et des produits du travail, a donc pour signification que c'est la société qui devient elle-même la porteuse du travail productif et que tous les individus deviennent productifs dès lors qu'ils participent de cette activité globale et que leur activité s'inscrit dans la division sociale du travail.

Avec la première analyse du procès de travail, située sur un plan strictement individuel, nous avions affaire à quelque chose dont le degré d'abstraction était tel que cela ne pouvait se passer dans aucune société ; avec cette seconde analyse, c'est l'inverse : nous avons affaire à quelque chose qui se passe dans toutes les sociétés et qui apparaît dès qu'il y a socialisation de l'activité de travail. Ces deux analyses du procès de travail et du travail productif sont donc abstraites et, à ce titre, insatis-

\footnotetext{
$5^{2}$ Ibid., p. 570.

53 Idem.

54 Idem.
} 
faisantes. On comprend que Marx soit pressé d'en venir au travail productif et au procès de travail dans la société dominée par le mode de production capitaliste. Car c'est là qu'intervient quelque chose de nouveau : à rebours de la socialisation dont on a vu qu'elle étendait la notion de travail productif en faisant de la société elle-même un travailleur global, il se produit au contraire, dans la production de type capitaliste, ce que Marx appelle « une sorte de rétrécissement» de la notion de travail productif. Cela est dû à la spécificité du procès capitaliste de production qui n'est pas seulement production de choses utiles, ni même seulement production de marchandises, c'est-àdire de choses porteuses de valeur, mais « essentiellement production de survaleur ».

Ici, le travailleur ne produit pas pour lui, il ne produit pas non plus pour un autre indéterminé et donc pour la société, il produit « pour le capital». Ce n'est donc ni le producteur individuel, ni la société qui définit ce qui est un travail productif, c'est le capital qui le définit comme tel. Pour qu'il soit productif et considéré comme tel, il ne suffit plus que le travailleur produise individuellement, ni qu'il produise socialement au sens où son activité prend part à l'activité sociale de production, « il faut qu'il produise de la survaleur $»$ : telle est maintenant la condition pour que son travail vaille comme du travail productif, et pour que lui-même soit reconnu comme travailleur productif. «Seul est productif le travailleur qui produit de la survaleur pour le capitaliste ou qui sert à la valorisation du capital55 ».

On a là le résultat de l'étude approfondie que Marx a faite à partir du début des années 1960 de la catégorie de travail productif et dont témoignent en particulier les Théories sur la plus-value. On sait toute l'importance que Marx accorde à Smith dans le chapitre IV des Théories, celui qu'il consacre au « travail productif et improductif ». Smith avait en effet écrit, dans La richesse des nations, que les « ouvriers productifs » sont ceux qui « reproduisent la valeur totale de leur consommation accompagnée d'un certain profit 56 ». Ce que Marx commente de la façon suivante : "ici le productive labourer est de toute évidence celui qui, non seulement reproduit la full value des moyens de subsistance contenus dans le salaire, mais qui la reproduit with a profit pour le capitaliste ». De sorte que Marx peut faire ici un éloge appuyé de Smith :

Smith, dit-il, a touché juste : sur le plan des concepts, il a épuisé la question. Un de ses plus grands mérites scientifiques est d'avoir défini le travail productif comme travail qui s'échange immédiatement contre le capital57.

Ce qui veut dire que « seul le travail qui produit du capital est productif », et donc le travail qui permet au capital de s'accroître et de se valoriser. La condition pour que cela ait lieu étant qu'il y ait échange entre le capital et la force de travail, et en

55 Idem.

${ }^{6}$ Cité par Karl Marx dans Théories sur la plus-value, (livre IV du « Capital »), t. 1, trad. G. Badia, Paris, Éditions sociales, 1974, p. 166.

57 Karl Marx, Théories sur la plus-value, op. cit., p. 167. 
l'occurrence un échange tel que la part de capital qui est versée comme salaire et qui est objectivée dans les moyens de travail soit « remplacée par plus de travail qu'elle n'en contient ». Cette conception du travail productif est celle que Marx reprend dans Le Capital, notamment au chapitre XIV où il écrit que « seul est productif le travailleur qui produit de la survaleur pour le capitaliste ou qui sert à la valorisation du capital $5^{8} \gg$. C'est aussi la conception que l'on trouve dans le Chapitre VI également nommé Chapitre inédit du Capital, dont la rédaction en 1864 est contemporaine de celle des Théories sur la plus-value: " est productif le travail qui crée immédiatement de la survaleur, c'est-à-dire qui valorise le capital59 ». Après quoi Marx ajoute encore ceci :

Travail productif n'est qu'une manière abrégée d'exprimer en leur ensemble le rapport et les modalités sous lesquelles figurent la capacité de travail et le travail dans le procès de production capitaliste. Si donc nous parlons de travail productif, nous disons travail socialement déterminé, travail incluant un rapport tout à fait déterminé entre le vendeur et l'acheteur de travail. Le travail productif s'échange directement contre de l'argent en tant que capital, autrement dit de l'argent qui est capital en soi, qui possède cette détermination de fonctionner comme capital et de faire face à la capacité de travail comme capital. Le travail productif est donc celui qui ne reproduit pour le travailleur que la valeur prédéterminée de sa capacité de travail en contrepartie de quoi, par son activité créatrice de valeur, il valorise le capital, oppose au travailleur en tant que capital les valeurs mêmes qu'il a créées. Le rapport spécifique entre le travail objectalisé et le travail vivant est que le premier en tant que capital fait du dernier du travail productif. Le produit spécifique du procès de production capitaliste, la survaleur, ne se crée que par échange avec le travail productif ${ }^{60}$.

Les catégories de travail et de travailleur productifs nous mettent ainsi en présence de l'une des multiples formes que prend la contradiction inhérente au mode de production capitaliste. D'une part, considérée de façon générale en tant que formation sociale, et donc comme toute autre forme de société, la société de type capitaliste étend les catégories de travail et de travailleurs productifs, et procède même plus qu'aucune autre société à cette extension : elle est une société qui accroît plus que nulle autre en son sein la division sociale du travail, qui rend les travaux individuels plus interdépendants que jamais et qui généralise les formes coopératives du travail, de sorte qu'en ce sens il n'y a plus aucun travail, même réduit à une tâche extrêmement partielle et limitée, qui ne soit actuellement ou potentiellement productif. Mais, d'autre part et dans le même temps, le même mode de production capitaliste procède à une restriction sans

$5^{8}$ Karl Marx, Le Capital, livre 1, op. cit., p. 570.

59 Karl Marx, Le Chapitre VI : Manuscrits de 1863-67. Le Capital, livre 1, trad. G. Cornillet, L. Prost et L. Sève, Paris, Éditions sociales, 2010, p. 212.

60 Ibid., p. 218-219. 
précédent des catégories de travail et de travailleur productifs, puisque c'est un mode de production qui engendre une forme de société au sein de laquelle ne sont plus reconnus comme productifs que les travaux qui accroissent le capital et en permettent la valorisation.

Cela est dû au fait que la société soumise au mode de production capitaliste est celle dans laquelle aucun individu ne peut devenir productif s'il n'a pas d'abord été échangiste ; l'entrée d'un individu dans le procès de production supposant qu'il ait d'abord vendu à autrui la marchandise qu'est sa propre force de travail : sans cet échange marchand préalable, l'individu n'entrerait pas dans le procès de production, et surtout, sans cet échange, ce procès ne pourrait pas être productif au sens proprement capitalistique du terme, c'est-à-dire qu'il ne pourrait pas être en même temps un procès de valorisation du capital consistant en ce que, en échangeant une part de capital avec la force de travail, « le capitaliste reçoit un quantum de temps de travail supérieur à celui qu'il a payé sous forme de salaire ${ }^{61} »$.

On peut donc dire, en d'autres termes, que les sociétés capitalistes, en restreignant et limitant comme elles le font le sens du travail productif, sont des formations sociales qui attestent par là même qu'en leur sein, ce n'est pas le procès de travail qui est porteur du lien social : le lien social y est assuré par l'échange marchand de la force de travail contre une part de capital sous la forme du salaire, et non pas directement par le procès de travail. En limitant le travail à la production et en restreignant le productif de la production aux seules activités qui valorisent le capital, les sociétés capitalistes montrent qu'en leur sein le lien social réside essentiellement non pas dans le travail et la production eux-mêmes, mais dans la vente et l'achat de la marchandise particulière qu'est la force de travail.

Pour finir, nous pouvons alors revenir au procès de travail tel que Marx le thématise au début du chapitre XIV du Capital. Dans le procès de travail considéré comme « appropriation individuelle des objets de la nature aux propres finalités vitales de l'individu ", ce dernier " a le contrôle de lui-même », et, " de même que le système de la nature fait de la tête et de la main un ensemble [individuel] unique, [de même] le procès de travail réunit travail cérébral et travail manuel ${ }^{62} »$. Ainsi se passent les choses aussi longtemps du moins qu'on considère le procès de travail comme « un procès purement individuel », ce qui n'est possible que par abstraction. Car le procès de travail est en réalité toujours un procès social : la socialisation du procès de travail introduit la division du travail, et en particulier la division entre travail manuel et travail cérébral: le sujet du procès de travail n'est plus l'individu, mais la société dans son ensemble, qualifiée par Marx de «travailleur global63» qui fait des individus des «organes » de lui-même en leur attribuant des fonctions séparées de travail matériel

\footnotetext{
${ }^{61}$ Karl Marx, Théories sur la plus-value, op. cit., p. 166.

62 Karl Marx, Le Capital, livre 1, op. cit., p. 569.

63 Ibid., p. 570.
} 
et de travail intellectuel. Cette séparation n'a rien de spécifique aux sociétés capitalistes, mais celles-ci poussent la division entre le travail de la main et le travail de la tête plus loin que n'importe quelle autre, en particulier en raison de la mobilisation productive de la science à laquelle elles procèdent. À quoi s'ajoute que, en tant que sociétés marchandes, elles déplacent le lien social du procès de travail vers l'échange, ce qu'elles font plus que nulle autre société marchande en soumettant l'échange luimême à la logique abstraite de la valorisation du capital, et donc en faisant de l'échange un moyen d'accroissement du capital. Le procès de travail est dès lors privé de toute possibilité de contrôle sur lui-même par lui-même : il est contrôlé et commandé de l'extérieur par le capital qui l'utilise et le mobilise comme procès de valorisation de lui-même.

S'esquisse alors, en contrepoint, la figure d'une société qui posséderait le contrôle d'elle-même de la même façon que le travailleur possède le contrôle de lui-même dans le procès de travail individuel, d'une société qui unirait «travail cérébral et travail manuel » comme « le système de la nature fait de la tête et de la main un ensemble unique ». Une telle société, réalisant ce que Sohn-Rethel appelait «l'unité sociale de la tête et de la main ${ }^{64}$ », est une société dans laquelle le procès de travail serait luimême et directement le véhicule du lien social, c'est une société dans laquelle le potentiel de socialisation portée par le travail serait pleinement développé en étant libéré de sa captation productive par l'échange entre capital et force de travail. C'est une société qui aurait soustrait au capital le monopole qu'il possède actuellement et qui est essentiellement le monopole de la décision portant sur ce qui est du travail productif et ce qui n'en est pas : mais retirer au capital ce monopole revient à le détruire en tant que capital.

\section{Conclusion}

Précisons encore, pour conclure, qu'il ne s'agit pas de transférer à une instance autre que le capital le pouvoir de décider de ce qui est travail productif et de ce qui ne l'est pas : il s'agit de contester la distinction même entre travail productif et improductif, au motif que cette opposition, partout où elle fonctionne, porte la marque du capital et qu'elle est à chaque fois un véhicule privilégié de la domination, non pas seulement du travail de la tête sur celui de la main, mais aussi du travail masculin (reconnu comme productif) sur le travail reproductif féminin (invisibilisé comme non productif), ou encore de la domination de l'espèce humaine (seule à être productive) sur le reste de la nature et l'ensemble des vivants non-humains. La domination du capital se manifeste très précisément à la puissance qui est la sienne de décider qui est productif et qui ne l'est pas, quel travail est productif et quel travail ne l'est pas. Il exerce cette puissance en contraignant les individus à l'échange de leur force de travail contre du capital,

64 Alfred Sohn-Rethel, « Travail intellectuel et travail manuel. Essai d'une théorie matérialiste », dans $L a$ pensée-marchandise, trad. L. Mercier, Bellecombe-en-Bauges, Éditions du Croquant, 2010, p. 146. 
c'est-à-dire en faisant d'eux de purs sujets séparés des conditions matérielles du procès de travail : le devenir productif d'une activité de travail sous le capital est en ce sens la destruction même de ce dont le procès de travail est en soi l'unité. Le procès de travail est une relation métabolique entre l'homme et la nature qui est rompue par l'enrôlement du procès de travail dans le procès productif comme procès de valorisation du capital. Cette rupture de l'unité métabolique du procès de travail est fondamentale : c'est là que se joue et se décide, dans l'antre secret de la production, la rupture du rapport métabolique entre les systèmes sociaux et les systèmes naturels ${ }^{65}$.

Notons que c'est d'ailleurs et très certainement de ce côté-là, celui de la prise en compte par Marx de la question du rapport entre systèmes sociaux et système naturel qu'il faut aller chercher la raison qui explique sa rupture avec le paradigme productif et son passage au paradigme du travail : cette dimension s'impose de plus en plus à Marx à partir de la fin des années 1850 et du début des années 1860, et elle s'accompagne de la reconnaissance de plus en plus nette non seulement de ce que le procès de travail, considéré aussi bien individuellement que socialement, est ce qui assure la médiation entre les deux types de systèmes (social et naturel), mais aussi de ce que les forces humaines de travail sont elles-mêmes des forces naturelles. D'où le fait que ces années sont aussi celles durant lesquelles Marx abandonne progressivement le terme de Arbeitsvermögen (capacité ou faculté de travail), encore dominant en 1857-1858 dans les Grundrisse, au profit de celui de Arbeitskraft (force de travail) qui s'impose définitivement dans le livre 1 du Capital. Ce changement terminologique manifeste et rend explicite l'appartenance de la force humaine de travail elle-même aux forces naturelles, de sorte que l'arraisonnement productif et même productiviste de cette force de travail par le capital peut désormais clairement être considéré comme une dénaturation dont les effets sur la nature elle-même sont potentiellement catastrophiques.

De sorte que la question ne peut finalement manquer de se poser de savoir à quel type de critique du capitalisme conduit notre distinction entre le paradigme de la production (propre à Marx 1) et le paradigme du travail (typique de Marx 2). Cette distinction, telle que nous la formulons, nous paraît donner raison à Moishe Postone quand il écrit que « la théorie critique du Marx de la maturité est une critique du travail sous le capitalisme, et non pas une critique du capitalisme du point de vue du travail66». Notre point de vue est également celui d'une critique du travail sous le

65 Voir Del Weston, The Political economy of global warming: The Terminal crisis, New York, Routledge, 2014, p. 65 : «Selon la théorie écologique de Marx, les humains vivent une relation "métabolique" avec la nature qui s'avère essentielle à leur survie. Cette relation prend la forme du travail, ce processus matériel par lequel les humains transforment les ressources brutes de la nature pour satisfaire leurs besoins matériels. [...] Le concept [de métabolisme, concept] clé de la critique écologique du capitalisme formulé par Marx rend compte d'une déconnexion entre les systèmes sociaux et le reste de la nature. La rupture métabolique est partie intégrante des rapports de production capitalistes » (traduction libre). C'est moi qui souligne.

66 Moishe Postone, Temps, travail et domination sociale. Une réinterprétation de la théorie critique de Marx, trad. O. Galtier, L. Mercier, Paris, Mille et Une Nuits, 2009, p. 42. 
capital, c'est-à-dire qu'il s'agit d'une critique de ce que devient le travail sous le capital, ou de ce que le capital fait au travail quand il l'enrôle et le soumet au processus de production et de valorisation de la valeur - une critique qui se formule donc non pas en fonction de ce que le travail serait en soi, en son essence (encore moins à l'aune de ce qu'il a été ou est supposé avoir été avant le capitalisme), mais, de façon projective, donc une critique qui se formule en fonction de ce que pourrait être le travail humain social $s$ 'il était libéré de son enrôlement par le procès de valorisation, c'est-àdire libéré de sa captation productive par le capital - ce qui permettrait aussi un retour à l'équilibre de la relation entre systèmes sociaux et système naturel. 